\title{
DE LA AGENDA POLÍTICA A LA RADIO EN SANTIAGO DE CUBA. ESTUDIO LONGITUDINAL DE TRANSFERENCIA TEMÁTICA.
}

Viviana Muñiz Zúñiga1: Universidad de Oriente, Cuba.

vivita@fch.uo.edu.cu

Rafael Ángel Fonseca Valido: Universidad de Oriente, Cuba.

fonseca@fch.uo.edu.cu

\section{RESUMEN}

Esta investigación presenta un análisis longitudinal en cuatro tiempos de la correlación entre la agenda mediática de la emisora local de Santiago de Cuba, CMKC Radio Revolución, y la agenda política. Tiene como objetivo general determinar la correlación existente entre la agenda mediática de la emisora CMKC y la agenda política en Santiago de Cuba durante los años 2014 y 2015. La investigación tiene como aporte que es la primera de su tipo en el país, al explicar la relación entre estas dos agendas en una provincia durante varias fases. El estudio está concebido desde una metodología cuantitativa, y se utilizan métodos como el Análisis- Síntesis y el Inductivo-Deductivo, y técnicas como el Análisis de Contenido, el coeficiente de Correlación por rangos de Spearman y la Observación participante. Con el análisis realizado se demuestra que existe una relación moderada y en ocasiones alta entre la agenda política y la agenda mediática estudiada, y que esta característica se mantiene estable en el tiempo analizado.

\section{PALABRAS CLAVE}

Agenda política- Agenda mediática- Correlaciones- Agenda Setting- Agenda Building.

\footnotetext{
1 Viviana Muñiz Zúñiga: Licenciada en Periodismo. Investiga desde el 2013 los procesos de construcción y establecimiento de la agenda mediática en Cuba; la implementación de las Política Informativa y Editorial y la construcción de la noticia en los medios cubanos.
} 


\title{
FROM THE POLITICAL AGENDA TO THE RADIO IN SANTIAGO DE CUBA. A LONGITUDINAL STUDIO OF THEMATIC TRANSFER.
}

\begin{abstract}
This piece of research presents a four-time longitudinal analysis of the correlation among the media agenda of the local radio station in Santiago de Cuba, CMKC Radio Revolution, and the political agenda. Its general objective is to determine the extant correlation among the media agenda of the radio station CMKC and the political agenda in Santiago de Cuba in the years 2014 and 2015. The contribution of this piece of research is that is the first of its type in the country, as it explains the relationship between these two agendas in a province during different phases. The studio has been conceived from a quantitative methodology, and methods like Analysis Synthesis and the Inductive-deductive are used as well as techniques such as Analysis of Content, correlation coefficient by Spearman ranges and participant Observation. With the analysis that was carried out it is demonstrated that there is a moderate, sometimes high relationship between the political agenda and the media agenda under study, and that this characteristic remains stable all through the time the analysis took place.
\end{abstract}

\section{KEY WORDS}

Political agenda - Media agenda - Correlations - Agenda Setting - Agenda Building.

\section{INTRODUCCIÓN}

Históricamente la radio ha servido como un vehículo de primer orden para el debate de las ideas y la expresión de la opinión pública. Inaugurada en Cuba en 1922, ya en los años 30 desempeñaba un papel básico en la expresión de la cultura y la identidad del cubano. Susceptible de influir y condicionar los criterios y creencias de los oyentes, este espacio entraba en los hogares apoderándose de todos los miembros a partir de una programación sumamente atractiva y enajenante, para cuyo disfrute no era necesario obviar las tareas habituales o compartir un espacio específico.

Campañas comerciales, radio novelas, dramatizados e incluso programas moralizadores anti-corrupción minaron los circuitos de las principales emisoras nacionales, que si bien respondían en sus mayoría a los intereses de la gran oligarquía cubana, nunca perdieron oportunidad para hacerse de grandes ratings de audiencia.

El triunfo de la Revolución cubana marcó entonces el inicio de un nuevo camino para los medios, y la radio no quedó inamovible. La masificación de la radio a partir de la creación de varias emisoras nacionales y provinciales, e incluso municipales le dio un nuevo contenido a esta prensa, convirtiéndose sus espacios en voceros principales de la opinión y del ejercicio del debate en nuestro país. 
En Cuba, como en el resto del mundo, los estudios sobre los emisores están ocupando un lugar privilegiado, dado por los nuevos roles que estos medios han demostrado jugar en la sociedad, como el llamado cuarto poder. Estudios de rutinas productivas, Newsmaking, gatekeepers, sociología de la producción, y Agenda Setting, etc. siguen invadiendo los escenarios investigativos comunicológicos ponderando el papel de estos como instrumentos de socialización, difusión de ideas y creación de significados.

Desde esta perspectiva, investigaciones que aborden a la radio como medio en la teoría del establecimiento temático (conocida también como Agenda Setting), y su relación con las agendas política y pública, no son abundantes en el país. Si bien la relación entre los políticos y los medios ha sido un objeto ampliamente estudiado desde la teoría de la Agenda Setting a nivel mundial (McCombs, 2006; López y Casero, 2014; Gautier y Ruiz, 2014), en Cuba ha sido un camino investigativo que no ha fructificado en gran medida.

La prensa cubana tiene como particularidad que responde a los intereses del pueblo, y está rectorada por una Política Informativa, como un conjunto de directrices que orienta el Partido Comunista de Cuba. Investigadores cubanos (Muñiz y Fonseca, 2015) han demostrado que la construcción de la agenda mediática es un proceso cuya estructura no se comporta de forma rígida, ni vertical; aunque inicialmente presentaba algunos rasgos de unilateralidad, sobre todo en la disposición de los temas. Por otra parte, la correlación entre esta agenda y la mediática se mantuvo alta (0.66) en un estudio desarrollado durante tres meses del 2013 en los medios provinciales santiagueros (Muñiz y Fonseca, 2015: 327). En tanto, la correlación entre la agenda mediática de la emisora CMKC con la agenda política fue moderada $(0,56)$. Por estas razones un estudio longitudinal de la agenda mediática de la emisora provincial de Santiago de Cuba, CMKC Radio Revolución, y su relación con la agenda política en el territorio, no solo constituye el primero de su tipo en el país, sino que permite sentar bases empíricas para posteriores hallazgos. En este sentido, se ha planteado como problema de investigación ¿Qué correlación existe entre la agenda mediática de la emisora CMKC y la agenda política en Santiago de Cuba durante los años 2014 y 2015 ?

\section{OBJETIVOS}

El objetivo general del trabajo es determinar la correlación existente entre la agenda mediática de la emisora CMKC y la agenda política en Santiago de Cuba durante los años 2014 y 2015.

Para ello se trazaron como tareas de investigación u objetivos específicos:

1. Determinar los objetos y atributos que componen la agenda política en la provincia durante los períodos de tiempo estudiados.

2. Caracterizar los objetos y atributos que figuran en la agenda mediática de la emisora CMKC durante las fases temporales del estudio.

3. Correlacionar objetos y atributos en la dimensión sustantiva y afectiva en las agendas mediática y política estudiadas.

\section{METODOLOGÍA}


En este estudio se plantea como hipótesis que la agenda mediática de la emisora CMKC y la agenda política en Santiago de Cuba poseen una correlación significativa, en tanto existe una transferencia temática desde la segunda hacia la primera. Esta particularidad se mantiene constante en el tiempo.

En el estudio se asumieron como variables la agenda mediática la emisora CMKC y la agenda política:

- $\quad$ Agenda mediática de la emisora CMKC: parte de la realidad que es atendida por el medio de prensa, y que se materializa en la selección, jerarquización y tratamiento de los acontecimientos que responden a sus intereses.

- Agenda política: Acciones que adoptan los gobiernos, parlamentos y las diferentes instituciones sociales que más tarde formarán parte desencadenante de debates, además de incluirse como temas destacados en la agenda de los medios y en la agenda pública.

Estas variables se operacionalizan de la siguiente forma:

- Objetos: Hechos de la realidad concreta cubiertos por los medios de comunicación, de forma constante o temporal. Como resultado de un estudio previo (Muñiz y Fonseca, 2015), se diferencian tres clasificaciones para los objetos en la agenda mediática:

o Tipo 1: Objetos con un alto grado de presencia: en esta categoría se incluyen aquellos cuya cantidad de menciones sobrepasa el $5 \%$ de las menciones totales.

o Tipo 2: Objetos con un moderado grado de presencia: esta categoría contiene los temas que sobrepasan el $2 \%$ de las menciones totales.

o Tipo 3: Objetos con un bajo grado de presencia: esta categoría contiene aquellos objetos cuyo porcentaje de menciones es inferior al $2 \%$ del total.

- Atributos: Elementos subjetivos que califican al objeto. Se dividen en dos dimensiones: una sustantiva, que incluye los rasgos que distinguen al objeto o los subtemas que se hallan implicados; y una afectiva, que es la valoración que se emite acerca de dichos temas, y puede ser Positiva, Neutra o Negativa.

Se asumió una perspectiva metodológica cuantitativa, de tipo longitudinal. Los métodos utilizados fueron el Análisis-síntesis y el Inductivo-deductivo. El primero de ellos posibilitó hacer una búsqueda y contraste de la bibliografía y los estudios existentes en Cuba acerca del fenómeno; en tanto, el segundo permitió generalizar elementos particulares de la agenda mediática de la emisora, y particularizar otros concernientes a la investigación sobre agenda política en el país. Como destacamos anteriormente, la radio es un medio poco estudiado en general en los estudios sobre agenda en Cuba, que se han enfocado principalmente en medios como la prensa plana y la televisión- local y nacional-.

En tanto, se emplearon técnicas como el análisis de contenido cuantitativo a los medios, cuyas especificidades abordaremos a continuación; la observación participante en las reuniones del Partido Comunista de Cuba con los medios, y la correlación por 
rangos de Spearman, cuya magnitud indica el grado de asociación entre las variables seleccionadas. Dada la existencia de diferentes softwares para determinar este valor, se decidió emplear el SPSS (Paquete Estadístico para Ciencias Sociales), versión 11.5. En el caso de las correlaciones especificaremos además de la interpretación y el valor, la cantidad de ítems correlacionables, porque el coeficiente puede dar un valor determinado que no sea indicativo de la situación matemática real.

Esto evidentemente ocurre porque las agendas son muy heterogéneas en su composición, y los ítems que están presentes en una no necesariamente están en otra. Por ello es importante tener en cuenta este aspecto, que permite ofrecer valoraciones más profundas del dato numérico que brinda el coeficiente. Este elemento permite ver también que la correlación es más fidedigna desde el punto de vista metodológico y procedimental, porque desde la perspectiva matemática cada correlación tiene un nivel de significación, que se determina utilizando el programa SPSS.

Para este estudio se diseñó un marco temporal especial que permitiese correlacionar las agendas mediática y política. Se decidió monitorearlas en el mismo período de tiempo, tomando como referente a la agenda política y comenzando una semana antes con la primera, ya que las orientaciones de coberturas pueden darse previamente (Muñiz y Fonseca, 2015). La Figura 1 permite visualizar en detalle los períodos delimitados para este estudio:

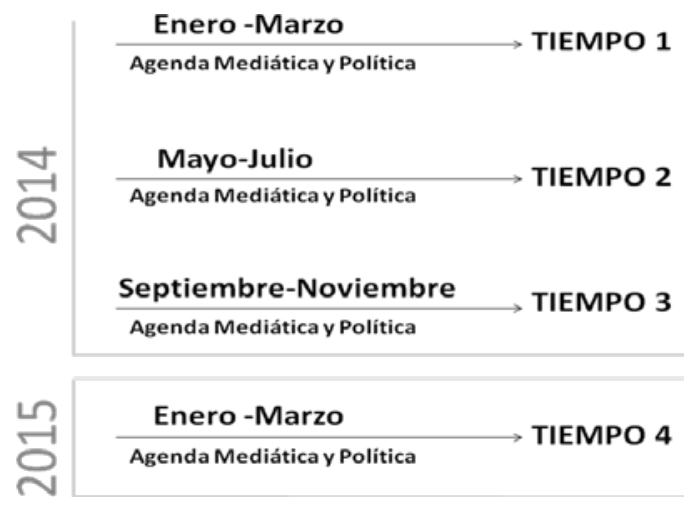

Figura 1 Marco temporal utilizado para la recogida de datos en el estudio. (Fuente: elaboración propia)

Para determinar la agenda mediática se realizó un análisis de contenido la emisora CMKC durante los cuatro períodos de estudio descritos, para el cual se tomó el total de trabajos periodísticos publicados en los programas informativos Reportes y Con el Sol.

En tanto, para caracterizar la agenda política se realizaron varios análisis de contenido a los documentos y reuniones del Partido Comunista de Cuba con la prensa. En el análisis de las agendas se estudiaron 27 objetos y 149 atributos en la dimensión sustantiva. 


\section{RESULTADOS Y DISCUSIÓN}

\subsection{Apuntes teóricos}

\subsubsection{La Agenda Setting como teoría}

Hablar de Agenda Setting es referirse al conjunto de temas seleccionados para formar parte de un índice o agenda, priorizados por diferentes actores sociales en condiciones disímiles.

En estado puro e incipiente esta escuela de pensamiento -que se inserta en los estudios de efectos a largo plazo- planteó que los medios de comunicación seleccionan de alguna manera un conjunto de temas resaltados, que serán traspasados por la agenda de los medios a la agenda del público. (McCombs, 2006) Por ello, el planteamiento esencial de estos postulados es la transferencia de relevancia temática desde una agenda hacia otra.

Esta perspectiva ha sido ampliada a lo largo de más de 40 años de estudios, hasta llegar a lo que consideramos hoy como Agenda Setting: un fenómeno complejo, en el que median diferentes actores sociales y factores externos e internos a los medios de comunicación y al público; y que permite en gran medida explicar la relación entre el sistema político, la prensa y la opinión pública.

Coincidimos con Maxwell McCombs, uno de los fundadores de esta escuela, cuando señala que la esencia de la teoría es la transferencia de relevancia de una agenda a otra (McCombs, 2006), y aunque plantea que esto ocurre desde los medios hacia la audiencia, agregamos que el fenómeno puede suceder también con respecto a la relación entre otras agendas (dígase política, escolar, religiosa, etc.), en diferentes direcciones y de manera integrada, como explicaremos posteriormente.

Con su surgimiento en el año 1968 se consolidó una metodología que permitiría demostrar y estudiar entre otros aspectos cómo los temas jerarquizados por los medios influían en los temas priorizados para la sociedad, y cómo el público se apropiaba de esos contenidos partiendo de condicionantes determinadas.

Estos procedimientos han ido evolucionando de manera que ya la agenda no se concibe solo desde un Análisis de Contenido, o una encuesta, sino desde modelos avanzados para evaluar la transferencia temática en red, o para evaluar la agenda pública en escenarios digitales.

Si bien diferentes autores la han enunciado de diversas maneras, cabe apuntar que el hecho de ser traducida al castellano ha motivado varias menciones del concepto. Se destacan algunas como "función del establecimiento de una agenda temática" (Agenda Setting function) o variaciones como "jerarquización de noticias" o "canalización periodística de la realidad" entre otras. En este trabajo se ha preferido adoptar el término Agenda Setting, por considerarlo ampliamente extendido y asimilado.

Las expresiones de "tematización de la realidad" o "fenómeno de tematización" han sido las utilizadas por el estructural-funcionalismo alemán para referirse también al mismo concepto. Para algunos autores (Dader, 1990 y Roda Fernández, 1989), la teoría de la Agenda Setting y la de la tematización consisten en un mismo proceso, aunque abordado desde una perspectiva mucho más profunda. 
Esta otra forma teorética de plantear la discusión sobre el fenómeno ha llevado a pensar a algún autor que se trataba de dos efectos diferentes, aunque relacionables. Aunque no pretendemos ahondar en esta discusión, sí consideramos pertinente esta aclaración para sustentar posteriores criterios.

En cuanto a las críticas realizadas a la teoría, puede decirse que existen varios textos (Vara, 2001; Casermeiro, 2004; Rodríguez, 2004; entro otros) que de una forma u otra sistematizan las recomendaciones hechas a las investigaciones. Entre las más sobresalientes figuran la diferencia semántica entre el establecimiento de la agenda y la tematización (Rodríguez, 2004:71), y la visión absolutista del papel de los actores políticos en el establecimiento de los temas en los medios (Rodríguez, 2004:72).

Además, se ha observado una insuficiente valoración del papel de las fuentes de información, y la preponderancia de las agendas electorales como principal objeto de estudio, lo que supone el establecimiento de condiciones metodológicas específicas para ese contexto (Rodríguez, 2004:71-74).

Autores como Roda Fernández (1989) y Raquel Rodríguez (2004) explican que a lo largo del desarrollo de las investigaciones- y consideramos que incluso en la actualidad- se le ha dado poca significación a los factores psicosociales que inciden en el establecimiento de la agenda mediática, lo que nos lleva a pensar en un reduccionismo en las llamadas variables influyentes; concebidas estrictamente para los escenarios en que han sido evaluados esos factores.

Estos estudiosos plantean la necesidad de incorporar variables como las expectativas de las personas acerca de los medios, y cómo a partir de estas se condiciona una actitud hacia un mayor o menos consumo de los mensajes. Sobre este particular hablaremos posteriormente en este capítulo.

Por otra parte, existe una contradicción de criterios en cuanto a la direccionalidad de la transferencia temática, pues el propio McCombs (2006) explica que el efecto de agendación se da desde los medios hacia el público, y no en sentido inverso.

Este criterio, que consideramos en gran medida absolutista, fue rebatido por David Weaver (1982), quien afirmó que es muy distinto afirmar que un grupo de temas influye en un grupo de personas, a que esos temas son realmente significativos para cada individuo en particular.

Aunque esta opinión de Weaver data de algunos años atrás, las metodologías de agenda han diferenciado entre los estudios de la influencia sobre una cantidad considerable de personas, de aquellos que versan sobre individuos particulares y su forma de asimilación de la agenda mediática.

Asimismo, concordamos con lo expresado por Raquel Rodríguez (2004) acerca de la exclusión de otras agendas mediáticas en la medición de la transferencia de temas sobre la agenda pública; porque sin lugar a dudas son igualmente determinantes otros consumos culturales -incluso mediáticos- que hace el individuo, y marcan su percepción acerca del mundo real.

De igual forma, se han hecho otros señalamientos metodológicos, relacionados con la corta duración las investigaciones, siendo pocas las que se extienden por un período mayor a un año (Petrone, 2009: 13); y la escasa inclusión de otras agendas y variables del mundo real (Petrone, 2009: 13; Rodríguez, 2004:72).

Cabe señalar en este sentido la falta de contextualización política y social de los 
estudios de agendas, y la interrelación propiamente dicha entre estas. Este particular nos ha hecho pensar en la importancia de la visión contextualizada de estos fenómenos, pues evidentemente muchos resultados planteados en sociedades capitalistas con un sistema político y social diferente al nuestro, no son aplicables a la realidad cubana.

En sentido general, la mayor parte de las observaciones realizadas a los estudios de agenda indican que existe una concentración en cuanto a las agendas analizadas, los métodos e instrumentos utilizados y evidentemente eso apunta hacia una estandarización metodológica. Si se estudian los planteamientos es posible ver que son menos los señalamientos de orden teórico -aunque no negamos que existan- que las cuestiones de orden empírico.

\subsubsection{Relación entre políticos y agenda mediática desde la Agenda Setting}

La Agenda Setting como proceso, integra a los políticos, los medios y el público en un escenario temático común, donde se definen los temas más importantes para la cobertura mediática.

Sobre este particular, el profesor chileno William Porath (2007, p. 43) explica que para los políticos, los medios de comunicación aparecen como el principal vehículo -más barato, rápido y masivo- para llegar al público con sus mensajes, desplazando en este rol al papel que alguna vez jugaron los partidos políticos.

Gans (1979) por su parte señala que la relación entre fuentes políticas y medios se asemeja a un tango, en el que es difícil decidir cuál de los actores lleva el paso en un determinado momento.

Lorenzo Gomis (1991) y Alsina (1993) se centran en esta línea de pensamiento, y explican la relación de intereses bilaterales entre agendas a partir la función del sistema político como productor-suministrador de hechos noticiosos. Señalan que las agendas, programas y planes de los órganos políticos están trazados en función de promover sus intereses; por tanto, se halla motivado por la información pública.

Sin embargo, el primero de esos autores refiere que las relaciones entre los políticos y los medios son más tensas de lo que podrían parecer por la intervención de dos factores distorsionadores:

- $\quad$ Selección cruel del material: de un $100 \%$ que es enviado a los medios, estos se ven obligados a reducirlo al menos al 10\%, por una cuestión básica de tiempo y espacio previstos.

- $\quad$ Presencia crítica del público, que se queja del exceso de información política en los medios, y prefiriendo otros temas en detrimento de los anteriores.

En este sentido, la cuarta fase de la teoría de la Agenda Setting se encarga precisamente de profundizar en las relaciones fuentes (políticas) en la conformación de la agenda de los medios. Conocida como Agenda Building, constituye una de las perspectivas más interesantes, dentro de la cual se ha investigado al presidente como agenda setter. Los estudios acerca del establecimiento de la adenda de los presidentes en las agendas mediática y pública han introducido nuevos elementos a la teoría, y en un sentido más amplio, han abierto nuevas brechas para desarrollar investigaciones que profundicen en la relación bilateral entre las agendas tanto en el 
nivel de objetos como en el de atributos.

Sin embargo los críticos han intentado desmitificar la visión de los líderes políticos como seleccionadores de temas de la agenda, porque se ha observado que la agenda las relaciones informales e interpersonales de los individuos modifican el proceso.

Disertaciones como la de Cook (et. al.1983) sostienen que en muchas ocasiones, de forma particular, los periodistas y los políticos intercambian información que beneficia los cambios o modificaciones de agendas. De esta manera no se podría hablar de la primacía de una agenda que influye en otra sino de una multitud de factores que interrelacionan las agendas (Van Aelst, Thesen, Walgrave, Vliegenthart, 2014; Gautier, y Ruiz, 2014).

Una tendencia renovada de los estudios sobre políticos y medios, sobre todo en los Estados Unidos, es aquella que investiga la agenda de grupos de presión y coaliciones, con respecto a la agenda de la Corte Suprema o al Congreso norteamericano (Goelzhauser y Vouvalis, 2015; Jackman, 2014; Van Aelst, Thesen, Walgrave, y Vliegenthart, 2014; Lindstädt, y Vander Wielen, 2014); algo que sin lugar a dudas indica que el campo de la llamada teoría de la Agenda Building es mucho más amplio de lo que realmente se ve, o se cree.

\subsection{Agenda política de la provincia Santiago de Cuba}

En sentido general, la jerarquización de los objetos en la agenda política durante los cuatro períodos estudiados no tuvo variaciones significativas. Recordemos que fueron analizados 27 objetos.

En la primera fase solo estuvieron presentes 19 de los 27 objetos evaluados en el estudio, como se muestra en la siguiente tabla.

\begin{tabular}{|c|c|c|c|c|c|c|c|c|}
\hline Objetos & Tiempo 1 & $\%$ & Tiempo 2 & $\%$ & Tiempo 3 & $\%$ & Tiempo 4 & $\%$ \\
\hline Actividades políticas, institucionales y sociales & 37 & $44,05 \%$ & 34 & $34,00 \%$ & 55 & $37,67 \%$ & 93 & $46,97 \%$ \\
\hline Acueducto & 0 & $0,00 \%$ & 0 & $0,00 \%$ & 2 & $1,37 \%$ & 2 & $1,01 \%$ \\
\hline Agricultura & 5 & $5,95 \%$ & 1 & $1,00 \%$ & 0 & $0,00 \%$ & 4 & $2,02 \%$ \\
\hline Ciencia y tecnología & 0 & $0,00 \%$ & 0 & $0,00 \%$ & 8 & $5,48 \%$ & 4 & $2,02 \%$ \\
\hline Comercio interior & 2 & $2,38 \%$ & 0 & $0,00 \%$ & 0 & $0,00 \%$ & 1 & $0,51 \%$ \\
\hline Comunicaciones & 2 & $2,38 \%$ & 1 & $1,00 \%$ & 2 & $1,37 \%$ & 7 & $3,54 \%$ \\
\hline Defensa civil & 1 & $1,19 \%$ & 1 & $1,00 \%$ & 2 & $1,37 \%$ & 3 & $1,52 \%$ \\
\hline Economía & 1 & $1,19 \%$ & 0 & $0,00 \%$ & 1 & $0,68 \%$ & 0 & $0,00 \%$ \\
\hline Educación & 0 & $0,00 \%$ & 11 & $11,00 \%$ & 0 & $0,00 \%$ & 3 & $1,52 \%$ \\
\hline Energía & 1 & $1,19 \%$ & 0 & $0,00 \%$ & 0 & $0,00 \%$ & 0 & $0,00 \%$ \\
\hline FAR-MININT & 2 & $2,38 \%$ & 3 & $3,00 \%$ & 3 & $2,05 \%$ & 3 & $1,52 \%$ \\
\hline Gastronomía & 0 & $0,00 \%$ & 0 & $0,00 \%$ & 0 & $0,00 \%$ & 0 & $0,00 \%$ \\
\hline Gobierno & 0 & $0,00 \%$ & 1 & $1,00 \%$ & 14 & $9,59 \%$ & 11 & $5,56 \%$ \\
\hline Higiene comunal & 5 & $5,95 \%$ & 5 & $5,00 \%$ & 6 & $4,11 \%$ & 1 & $0,51 \%$ \\
\hline Historia & 9 & $10,71 \%$ & 23 & $23,00 \%$ & 16 & $10,96 \%$ & 34 & $17,17 \%$ \\
\hline Hotelería y recreación & 0 & $0,00 \%$ & 1 & $1,00 \%$ & 1 & $0,68 \%$ & 1 & $0,51 \%$ \\
\hline Industria & 6 & $7,14 \%$ & 8 & $8,00 \%$ & 5 & $3,42 \%$ & 7 & $3,54 \%$ \\
\hline Justicia & 0 & $0,00 \%$ & 0 & $0,00 \%$ & 0 & $0,00 \%$ & 0 & $0,00 \%$ \\
\hline Medio ambiente & 1 & $1,19 \%$ & 5 & $5,00 \%$ & 0 & $0,00 \%$ & 2 & $1,01 \%$ \\
\hline Organizaciones políticas y de masas & 1 & $1,19 \%$ & 0 & $0,00 \%$ & 0 & $0,00 \%$ & 1 & $0,51 \%$ \\
\hline Política & 4 & $4,76 \%$ & 1 & $1,00 \%$ & 6 & $4,11 \%$ & 4 & $2,02 \%$ \\
\hline Problemas sociales & 1 & $1,19 \%$ & 1 & $1,00 \%$ & 7 & $4,79 \%$ & 1 & $0,51 \%$ \\
\hline Salud pública & 1 & $1,19 \%$ & 0 & $0,00 \%$ & 1 & $0,68 \%$ & 5 & $2,53 \%$ \\
\hline Servicios e instituciones estatales & 1 & $1,19 \%$ & 2 & $2,00 \%$ & 3 & $2,05 \%$ & 3 & $1,52 \%$ \\
\hline Trabajo y seguridad social & 1 & $1,19 \%$ & 0 & $0,00 \%$ & 1 & $0,68 \%$ & 1 & $0,51 \%$ \\
\hline Transporte & 0 & $0,00 \%$ & 0 & $0,00 \%$ & 1 & $0,68 \%$ & 3 & $1,52 \%$ \\
\hline Vivienda, construcción y patrimonio & 3 & $3,57 \%$ & 2 & $2,00 \%$ & 12 & $8,22 \%$ & 4 & $2,02 \%$ \\
\hline Total & 84 & $100,00 \%$ & 100 & $100,00 \%$ & 146 & $100,00 \%$ & 198 & $100,00 \%$ \\
\hline
\end{tabular}

Tabla 1 Objetos de la agenda política durante los cuatro períodos estudiados

Los primeros cinco temas de esta agenda en la primera fase fueron las Actividades políticas, institucionales y sociales $(44,05 \%)$, la Historia $(10,71 \%)$, la Industria $(7,14 \%)$, 
y la Agricultura y la Higiene comunal (5,95\% en ambos casos).

En la segunda fase aparecieron 16 objetos de los 27 analizados en esta investigación, manteniéndose Actividades políticas, institucionales y sociales en primera posición (34,00\%). En tanto la Historia ocupó el segundo puesto (23,00\%), y la Educación el tercero $(11,00 \%)$. A estos les siguieron la Industria $(8,00 \%)$, y la Higiene comunal y el Medio ambiente, ambos con un 5,00\% de las menciones totales.

Hasta aquí puede verse que los temas no poseen un alto grado de variación, según el período del año, correspondiente a los meses de enero a abril, y de mayo a agosto; sin embargo a partir de septiembre la agenda comienza a presentar ligeras variaciones.

Durante el tercer período de tiempo el objeto Actividades políticas, institucionales y sociales estuvo en la primera posición $(37,67 \%)$, seguido nuevamente de la Historia $(10,96 \%)$. Sin embargo en este caso se introdujeron en los primeros lugares otros temas como el Gobierno (9,59\%), la Vivienda, construcción y patrimonio $(8,22 \%)$ y la Ciencia y tecnología (5,48\%). En esta ocasión solo aparecieron 19 objetos de los 27 evaluados -como sucedió en la primera fase-.

La variación temática en este período puede deberse en gran medida a las proyecciones del gobierno y el Partido por el 500 aniversario de la fundación de la Villa, que se celebró en julio de 2015. Aquí podemos ver cómo la agenda puede cambiar en dependencia del contexto, pues en este caso se comenzaron a priorizar temas relacionados con el Gobierno y la Vivienda, dos aspectos esenciales para celebrar el aniversario.

Por último, en la cuarta fase del estudio se abordaron 23 objetos de 27 estudiados, entre los cuales se priorizaron las Actividades políticas, institucionales y sociales $(46,97 \%)$, la Historia $(17,17 \%)$, el Gobierno $(5,56 \%)$, y la Industria y las Comunicaciones, ambas con un 3,54\% de las menciones totales.

En esta agenda hubo dos objetos de los 27 evaluados que no aparecieron en ningún período: la Gastronomía y la Justicia. De ellos, el segundo fue el único que no estuvo en la agenda mediática general durante la primera fase.

En este sentido puede decirse que las dos prioridades temáticas en la agenda política son las Actividades políticas, institucionales y sociales- entiéndase actos, marchas, recorridos, entre otras- y la Historia -conmemoraciones y efemérides-. Como se verá más adelante, estos objetos no figuran en la agenda pública, por lo que no constituyen un asunto de interés social, y sí político. Además, las prioridades temáticas en esta agenda se mantienen estables en el tiempo, aunque evidentemente cuando un tema sube de rango en la agenda, otro tiene que descender. Otro elemento a destacar es que no estuvieron presentes en la agenda política los 27 objetos que han sido evaluados en la investigación, fluctuando la cantidad de apariciones por períodos de tiempo.

Por otra parte, se evaluaron 149 atributos en la dimensión sustantiva. En la primera fase del estudio solo estuvieron presentes en esta agenda 28 de los 149 atributos analizados en el estudio. De ellos se priorizaron las Reuniones (28,57\%), las Efemérides y conmemoraciones (9,52\%), la Zafra azucarera $(7,14 \%)$, los Eventos $(5,95 \%)$, el Cuadro higiénico (5,95\%), los Actos y abanderamientos $(4,76 \%)$, y los Recorridos, la Producción de alimentos agrícolas y la Producción y venta de materiales de la construcción, con un 3,57\% respectivamente. 
En el segundo período de tiempo la jerarquización se mantuvo de forma similar, con las Efemérides y conmemoraciones al frente $(19,00 \%)$, seguidas por las Reuniones $(15,00 \%)$, y los Eventos (14,00\%). Las Graduaciones (10,00\%) y la Zafra azucarera $(5,00 \%)$ les sucedieron en prioridad. En este caso solo encontramos 26 del total de atributos analizados.

Como se observa entre estos dos períodos no existe una diferenciación notable, porque los dos primeros puestos estuvieron ocupados por los mismos atributos en orden diferente. Por supuesto, ambos se corresponden al objeto Actividades políticas, institucionales y sociales, que -como ya vimos- encabeza la agenda política. Sin embargo los atributos más mencionados no se corresponden con los temas priorizados en estos períodos de tiempo.

Sin embargo, esto no sucede en la tercera fase el estudio, en la cual coinciden los atributos en la dimensión sustantiva con los objetos que encabezaron la agenda temática. En ese sentido encontramos en los primeros lugares a las Reuniones $(15,07 \%)$, los Eventos (13,7\%), las Efemérides y conmemoraciones (7,53\%), el 500 Aniversario (6,85\%), y las Investigaciones, las Medidas higiénicas y el Programa de la vivienda, con un $4,11 \%$ respectivamente. En este período se encontraron 38 atributos de 149 tenidos en cuenta para la investigación.

En caso similar se encuentra la agenda de atributos sustantivos en la cuarta y última fase, en la que estuvieron 45 atributos de los evaluados, entre los que estuvieron como primeros las Reuniones (33,33\%), las Efemérides y conmemoraciones (14,14\%), los Recorridos (4,55\%), y el 500 Aniversario y las Visitas, ambas con un 4,04\%.

Estos datos confirman lo que planteamos anteriormente acerca de la estabilidad temporal de los temas en la agenda política, lo que podría indicar que estas prioridades se mantienen estancadas.

En el caso de los atributos en la dimensión afectiva positiva, durante el primer período estudiado solo tuvieron menciones positivas los objetos Actividades políticas, institucionales y sociales $(66,67 \%)$, la Política $(16,67 \%)$, y las Comunicaciones, los Servicios e instituciones estatales y el Trabajo y seguridad social, con un 5,56\% de las menciones respectivamente.

Por otra parte, las Actividades políticas, institucionales y sociales (32,56\%), la Historia $(27,91 \%)$, la Educación $(16,28 \%)$, FAR-MININT (6,98\%) y los Servicios e instituciones estatales $(4,65 \%)$, son los objetos que poseían mayor cantidad de menciones positivas en la segunda fase.

Obsérvese que en estos dos períodos- al igual que sucede con siguientes- el objeto que definitivamente posee mayor cantidad de menciones positivas en el mismo que encabeza la agenda de objetos: las Actividades políticas, institucionales y sociales, seguido de la Historia. En los casos que veremos a continuación ocurre de manera similar, correspondiéndose con las prioridades temáticas de la agenda política de objetos.

En ese sentido, durante el tercer período encabezaron las menciones positivas los objetos Actividades políticas, institucionales y sociales $(37,70 \%)$, el Gobierno $(21,31 \%)$, la Historia $(18,03 \%)$, la Ciencia y tecnología $(8,20 \%)$, la Política $(6,56 \%)$ y los Problemas sociales (3,28\%).

Por último, en la cuarta fase solo fueron mencionados de forma positiva los objetos 
Historia (52,94\%), las Actividades políticas, institucionales y sociales $(17,65 \%)$, las Comunicaciones $(17,65 \%)$, la Vivienda, construcción y patrimonio $(5,88 \%)$ y la Defensa civil (5,88\%).

La deficiente presencia de menciones positivas en la agenda política se explica cuando observamos la cantidad de menciones neutras en esta agenda durante las fases, que superan con creces a las primeras; indicando que la agenda política se mantiene en la mayor parte de las ocasiones en un plano neutral.

Otro elemento a denotar en este sentido es que la Agenda Política está compuesta principalmente por indicaciones de coberturas, acerca de las cuales no se emiten criterios, más allá de que en algunos casos se reitere la necesidad e importancia de que sean atendidas por lo medios- como se constató a través de la Observación participante a las reuniones del Partido Comunista de Cuba con los medios-.

Volviendo a las menciones neutras, puede decirse que en la primera fase los objetos más resaltados en este sentido fueron las Actividades políticas, institucionales y sociales $(37,88 \%)$, la Historia $(13,64 \%)$, la Industria (9,09\%), y la Agricultura y la Higiene comunal, ambas con un $7,58 \%$ de las menciones para esta dimensión en el tiempo 1.

En el segundo período la mayor cantidad de menciones neutras fue para los objetos Actividades políticas, institucionales y sociales (35,09\%), Historia (19,30\%), Industria $(14,04 \%)$, e Higiene comunal y Medio ambiente, ambas con un $8,77 \%$ de las menciones. Obsérvese la similitud en los temas priorizados según estas menciones, y cómo se igualan los dos primeros con aquellos que encabezan la agenda de objetos. Esto sucede igualmente en los dos períodos siguientes.

Durante la tercera fase la dimensión afectiva neutral se mantuvo alta para el objeto Actividades políticas, institucionales y sociales $(37,65 \%)$, que posee un porcentaje mucho mayor al objeto que ocupa el segundo lugar: Vivienda, construcción y patrimonio (12,94\%) e Higiene comunal, que tiene el tercero con un 7,06\% de las menciones. Le siguen en el cuarto lugar Historia, Industria y Problemas sociales, con un $5,88 \%$ de las menciones respectivamente.

Por último, durante el último período los objetos Actividades políticas, institucionales y sociales $(49,72 \%)$, Historia (13,81\%), Gobierno $(6,08 \%)$ e Industria $(3,87 \%)$, tuvieron la mayor cantidad de menciones neutras, con una gran similitud entre estos y los temas priorizados en la agenda política en las fases analizadas.

Por otra parte, es preciso destacar que la agenda política no tuvo menciones negativas en ninguno de los cuatro períodos estudiados. Consideramos que esta es la principal condicionante para explicar el resultado, porque la realidad es mucho más rica, y la preocupación de las autoridades políticas por problemas que fustigan la realidad santiaguera pudo comprobarse a través de la Observación participante en las reuniones del Partido Comunista de Cuba con los medios. El problema radica en que a la hora de orientar las coberturas no se enfatiza en si es bueno o malo el hecho, porque simplemente los funcionarios emiten las orientaciones y dejan a los periodistas decidir qué enfoque darle.

A modo de resumen puede decirse que los objetos en la agenda política se mantienen estables en el tiempo, y que las prioridades temáticas en esta agenda oscilan entre las actividades como marchas, eventos, reuniones, entre otras, y otros temas como la 
Historia, la Vivienda y la Higiene, como puede observarse en la Figura siguiente:

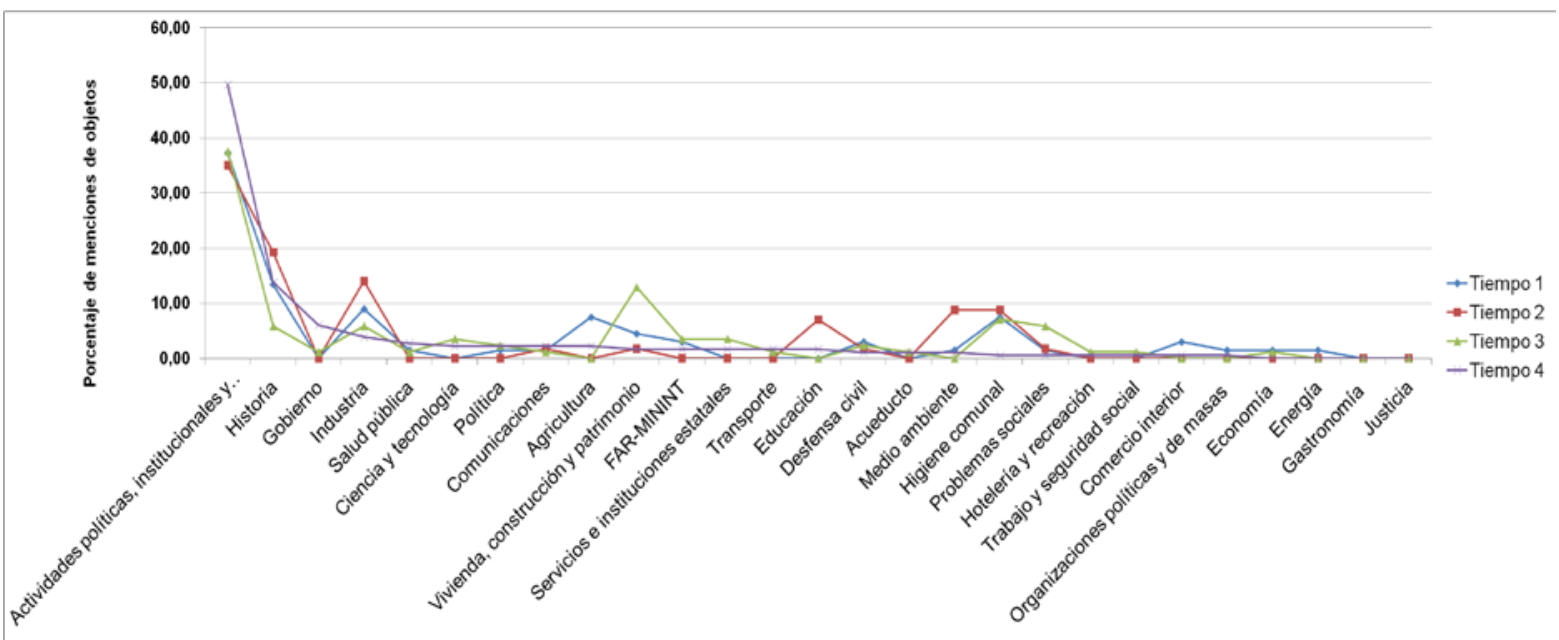

Figura 2 Permanencia de objetos (porcentaje) en la agenda política durante las fases de estudio. (Fuente: elaboración propia)

\subsection{Agenda mediática de la emisora CMKC y correlación con la agenda política}

La agenda mediática de la emisora en el primer período estudiado tenía 26 objetos de los 27 totales evaluados en la investigación. Así puede observarse en la tabla que mostramos a continuación:

\begin{tabular}{|c|c|c|c|c|c|c|c|c|}
\hline Objetos & Tiempo 1 & $\%$ & Tiempo 2 & $\%$ & Tiempo 3 & $\%$ & Tiempo 4 & $\%$ \\
\hline Actividades políticas, institucionales y sociales & 37 & $44,05 \%$ & 34 & $34,00 \%$ & 55 & $37,67 \%$ & 93 & $46,97 \%$ \\
\hline Acueducto & 0 & $0,00 \%$ & 0 & $0,00 \%$ & 2 & $1,37 \%$ & 2 & $1,01 \%$ \\
\hline \begin{tabular}{|l|} 
Agricultura \\
\end{tabular} & 5 & $5,95 \%$ & 1 & $1,00 \%$ & 0 & $0,00 \%$ & 4 & $2,02 \%$ \\
\hline Ciencia y tecnología & 0 & $0,00 \%$ & 0 & $0,00 \%$ & 8 & $5,48 \%$ & 4 & $2,02 \%$ \\
\hline Comercio interior & 2 & $2,38 \%$ & 0 & $0,00 \%$ & 0 & $0,00 \%$ & 1 & $0,51 \%$ \\
\hline Comunicaciones & 2 & $2,38 \%$ & 1 & $1,00 \%$ & 2 & $1,37 \%$ & 7 & $3,54 \%$ \\
\hline \begin{tabular}{|l} 
Defensa civil \\
\end{tabular} & 1 & $1,19 \%$ & 1 & $1,00 \%$ & 2 & $1,37 \%$ & 3 & $1,52 \%$ \\
\hline Economía & 1 & $1,19 \%$ & 0 & $0,00 \%$ & 1 & $0,68 \%$ & 0 & $0,00 \%$ \\
\hline Educación & 0 & $0,00 \%$ & 11 & $11,00 \%$ & 0 & $0,00 \%$ & 3 & $1,52 \%$ \\
\hline Energía & 1 & $1,19 \%$ & 0 & $0,00 \%$ & 0 & $0,00 \%$ & 0 & $0,00 \%$ \\
\hline FAR-MININT & 2 & $2,38 \%$ & 3 & $3,00 \%$ & 3 & $2,05 \%$ & 3 & $1,52 \%$ \\
\hline Gastronomía & 0 & $0,00 \%$ & 0 & $0,00 \%$ & 0 & $0,00 \%$ & 0 & $0,00 \%$ \\
\hline Gobierno & 0 & $0,00 \%$ & 1 & $1,00 \%$ & 14 & $9,59 \%$ & 11 & $5,56 \%$ \\
\hline Higiene comunal & 5 & $5,95 \%$ & 5 & $5,00 \%$ & 6 & $4,11 \%$ & 1 & $0,51 \%$ \\
\hline Historia & 9 & $10,71 \%$ & 23 & $23,00 \%$ & 16 & $10,96 \%$ & 34 & $17,17 \%$ \\
\hline Hotelería y recreación & 0 & $0,00 \%$ & 1 & $1,00 \%$ & 1 & $0,68 \%$ & 1 & $0,51 \%$ \\
\hline Industria & 6 & $7,14 \%$ & 8 & $8,00 \%$ & 5 & $3,42 \%$ & 7 & $3,54 \%$ \\
\hline Justicia & 0 & $0,00 \%$ & 0 & $0,00 \%$ & 0 & $0,00 \%$ & 0 & $0,00 \%$ \\
\hline Medio ambiente & 1 & $1,19 \%$ & 5 & $5,00 \%$ & 0 & $0,00 \%$ & 2 & $1,01 \%$ \\
\hline Organizaciones políticas y de masas & 1 & $1,19 \%$ & 0 & $0,00 \%$ & 0 & $0,00 \%$ & 1 & $0,51 \%$ \\
\hline Política & 4 & $4,76 \%$ & 1 & $1,00 \%$ & 6 & $4,11 \%$ & 4 & $2,02 \%$ \\
\hline Problemas sociales & 1 & $1,19 \%$ & 1 & $1,00 \%$ & 7 & $4,79 \%$ & 1 & $0,51 \%$ \\
\hline Salud pública & 1 & $1,19 \%$ & 0 & $0,00 \%$ & 1 & $0,68 \%$ & 5 & $2,53 \%$ \\
\hline Servicios e instituciones estatales & 1 & $1,19 \%$ & 2 & $2,00 \%$ & 3 & $2,05 \%$ & 3 & $1,52 \%$ \\
\hline Trabajo y seguridad social & 1 & $1,19 \%$ & 0 & $0,00 \%$ & 1 & $0,68 \%$ & 1 & $0,51 \%$ \\
\hline Transporte & 0 & $0,00 \%$ & 0 & $0,00 \%$ & 1 & $0,68 \%$ & 3 & $1,52 \%$ \\
\hline Vivienda, construcción y patrimonio & 3 & $3,57 \%$ & 2 & $2,00 \%$ & 12 & $8,22 \%$ & 4 & $2,02 \%$ \\
\hline Total & 84 & $100,00 \%$ & 100 & $100,00 \%$ & 146 & $100,00 \%$ & 198 & $100,00 \%$ \\
\hline
\end{tabular}

Tabla 2 Agenda de objetos de la emisora CMKC Radio Revolución durante las cuatro fases del estudio 
De ellos figuran como objetos con un alto grado de presencia las Actividades políticas, institucionales y sociales $(15,73 \%)$, la Salud pública $(9,98 \%)$, la Historia $(9,74 \%)$, las Comunicaciones (6,57\%), la Agricultura (6,46\%), los Servicios e instituciones estatales $(6,22 \%)$ y los Problemas sociales $(5,16 \%)$. Esta agenda tuvo una correlación Moderada $(0,423)$ con la agenda política, con 19 elementos correlacionables, algo que resulta significativo si revisamos que la segunda agenda precisamente posee 19 objetos. Esto quiere decir que todos los objetos de la agenda política estaban presentes en la mediática, aunque no en el mismo orden de aparición.

En el segundo período de tiempo la agenda de CMKC tuvo 27 objetos en total, de los cuales estuvieron en primer lugar la Historia $(10,61 \%)$, la Salud pública $(9,36 \%)$, las Actividades políticas, institucionales y sociales $(8,49 \%)$, la Agricultura $(6,37 \%)$, la Educación (6,12\%), los Servicios e instituciones estatales (5,62\%) y los Problemas sociales (5,37\%). En cuanto a la correlación con la agenda política, esta agenda mostró un resultado Moderado (0,549), con 16 elementos correlacionables. En este caso sucede lo mismo que en período anterior: todos los objetos de la agenda política están presentes en la mediática, pero no en el mismo orden de prioridad.

Durante la tercera fase del estudio la agenda de la emisora se mantuvo con 27 objetos, entre los cuales se destacan las Actividades políticas, institucionales y sociales $(12,21 \%)$, la Salud pública $(11,43 \%)$, la Agricultura $(10,64 \%)$, la Historia $(9,18 \%)$ y los Servicios e instituciones estatales $(6,74 \%)$. Véase que las prioridades temáticas continúan manteniéndose estables en el tiempo. En esta etapa la correlación con la agenda política se mantuvo Moderada $(0,427)$, con 19 ítems correlacionables -la misma cantidad que poseía la agenda política-.

Por último, en la cuarta etapa la agenda de CMKC tuvo 26 objetos, entre los que se ubicaron en primer lugar las Actividades políticas, institucionales y sociales $(20,60 \%)$, la Salud pública $(12,99 \%)$, la Historia $(8,96 \%)$, la Industria $(6,42 \%)$, las Organizaciones políticas y de masas $(5,67 \%)$ y las Comunicaciones $(5,52 \%)$.

La correlación de esta agenda con la agenda política fue Moderada $(0,56)$, con 23 ítems correlacionables- el total de los que posee la agenda política-. Este resultado es similar al obtenido por los autores Muñiz y Fonseca (2015:327), el cual muestra una relación Moderada $(0,56)$ entre ambas agendas, obtenida en un estudio transversal durante el año 2013. Por otra parte, si observamos la Figura siguiente vemos cómo los temas se mantienen constantes a lo largo del tiempo en cuanto al rango que se le otorga en la agenda -lo que no excluye que esto sufra variaciones-. 


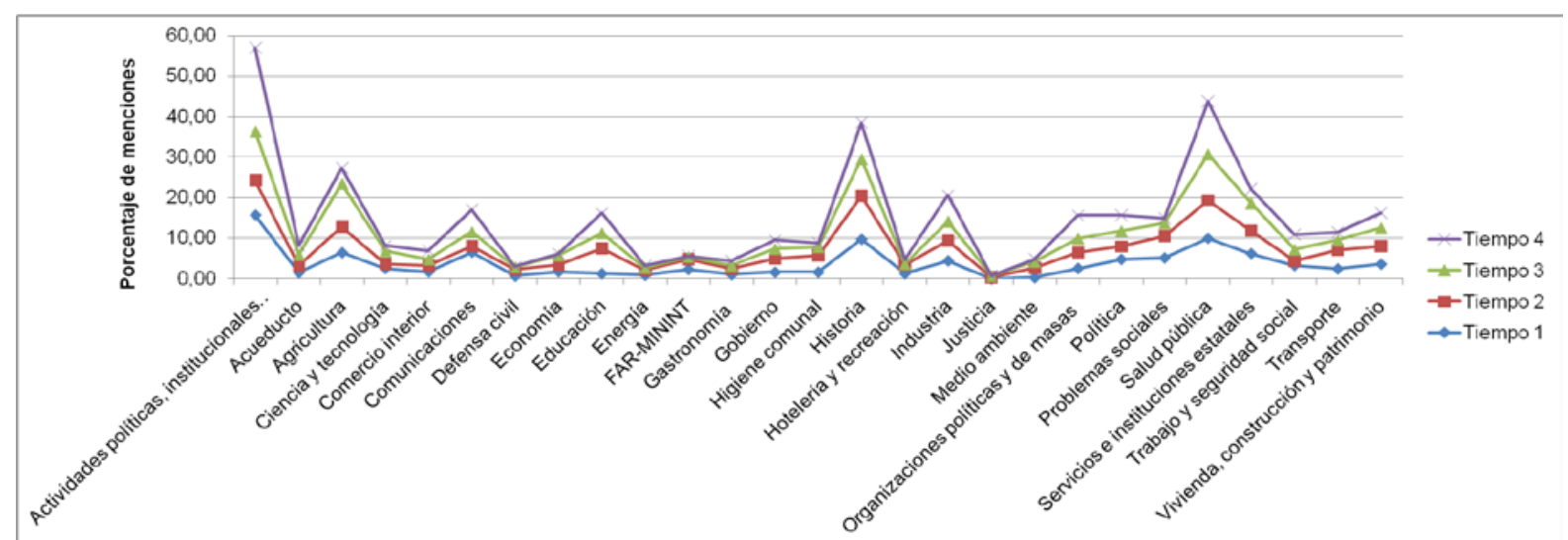

Figura 3 Permanencia de objetos (porcentaje) en la agenda de la emisora CMKC durante las fases de estudio. (Fuente: elaboración propia)

En cuanto a la dimensión sustantiva de los atributos, puede decirse que de 149 elementos en la agenda de CMCK solo hubo 98 en la primera fase. En este sentido, los atributos con más menciones fueron las Reuniones (9,62\%), las Efemérides y conmemoraciones y las Celebraciones y trabajo de la prensa $(5,28 \%$ cada uno), la Atención médica a la población $(4,23 \%)$, los Homenajes $(3,87 \%)$, la Prevención de enfermedades (3,29\%), los Eventos, la Zafra azucarera y los Servicios estatales a la población, con un 3,17\% respectivamente, y la Producción de alimentos agrícolas $(3,05 \%)$. Estos atributos, que concuerdan en gran medida con los objetos que encabezan la agenda de la emisora en esta etapa, tuvieron una correlación Baja $(0,379)$ con la agenda política, con solo 25 elementos correlacionables.

En ese sentido es importante denotar que si de 28 ítems posibles en la agenda política, hay 23 que aparecen en la agenda mediática de la emisora, entonces la mayor parte de los atributos de la primera están en la segunda. Por ello, aunque el valor de la correlación no sea elevado, el análisis cualitativo permite llegar a la conclusión de que sí existe una transferencia temática- como ocurre en los tres casos que veremos a continuación-.

Durante el segundo período la agenda mediática de la emisora tuvo 109 atributos sustantivos, entre los cuales se destacaron por la cantidad de menciones los Eventos $(6,12 \%)$, las Efemérides y conmemoraciones (5,74\%), los Homenajes $(4,62 \%)$, el Funcionamiento de organizaciones y tareas $(3,87 \%)$, la Atención médica a la población $(3,50 \%)$, las Instituciones estatales y la Lucha contra el mosquito $(2,87 \%$ cada una). Obsérvese como a pesar de tener una agenda de objetos constante en el tiempo, la agenda de atributos sustantivos es mucho más dinámica si evaluamos la variación de los temas en los primeros lugares. Por otra parte, la correlación de esta agenda con la política fue Moderada $(0,404)$, con 26 ítems correlacionables, que se corresponden con los que aparecen en la segunda agenda.

En la tercera fase del estudio la agenda de CMKC tuvo 105 atributos en la dimensión sustantiva, entre los que se encontraron en los primeros lugares los Eventos (7,32\%), las Efemérides y conmemoraciones (6,93\%), la Atención médica a la población $(4,59 \%)$, la Cosecha cafetalera y tabacalera $(3,91 \%)$, la Prevención de enfermedades $(3,61 \%)$, las Instituciones estatales y los Servicios estatales a la población (3,32\%). En este caso la correlación con la agenda política fue Baja $(0,323)$, con 35 elementos 
correlacionables. Esto resulta significativo si tenemos en cuenta que la segunda posee solo 38 atributos sustantivos en la agenda.

Por último, en la cuarta etapa de la investigación la agenda de la emisora tuvo solo 88 atributos, entre los que se ubicaron en los primeros lugares las Reuniones $(11,04 \%)$, los Eventos (7,61\%), las Efemérides y conmemoraciones (7,31\%), la Atención médica a la población $(5,82 \%)$, el Funcionamiento de organizaciones y tareas $(5,37 \%)$, las Celebraciones y trabajo de la prensa $(4,18 \%)$ y la Zafra azucarera $(3,43 \%)$. La correlación con la agenda política fue Baja $(0,314)$, con 32 ítems correlacionables, de 45 que tenía esta agenda.

Puede resumirse de este análisis que la presencia de los atributos en la dimensión sustantiva es menos constante en el tiempo que los objetos, pero no deja de observarse. Además, aunque los valores de las correlaciones son bajos, la cantidad de ítems correlacionables revela que en efecto todos o casi todos los atributos que están en la agenda política igualmente figuran en la mediática, aunque no en el mismo orden de prioridad.

En cuanto a la dimensión afectiva de los atributos, puede decirse que en la primera etapa la agenda de CMKC tuvo 25 objetos con menciones positivas, entre los cuales se destacan las Actividades políticas, institucionales y sociales $(15,86 \%)$, la Historia $(13,06 \%)$, la Salud pública $(9,33 \%)$, los Servicios e instituciones estatales $(7,84 \%)$, la Agricultura (7,28\%), las Comunicaciones $(6,34 \%)$, la Política $(5,60 \%)$ y la Industria $(5,22 \%)$.

La correlación de esta agenda con la política en esta dimensión fue Moderada $(0,447)$, con 5 elementos correlacionables- los existentes en la segunda agenda-, lo que indica que coinciden las menciones positivas de los objetos en ambas agendas.

En el segundo período hubo 26 objetos con menciones positivas. De ellos ocupaban los primeros lugares la Historia $(13,90 \%)$, las Actividades políticas, institucionales y sociales $(9,27 \%)$, la Salud pública $(8,11 \%)$, la Industria $(7,14 \%)$, los Servicios e instituciones estatales y la Educación (6,76\% cada uno), y la Agricultura (6,37\%). Obsérvese que en el primer caso, en este y en otros que veremos a continuación, los objetos con más menciones positivas coinciden con los priorizados por la agenda de la emisora. La correlación con la agenda política fue Alta $(0,693)$ con 10 elementos correlacionables -igual cantidad que los que aparecen en esta agenda-.

Por otra parte, en el tercer período hubo 26 objetos con menciones positivas, y de ellos los primeros fueron la Historia, las Actividades políticas, institucionales y sociales y la Agricultura (12,19\% cada uno), la Salud pública (9,87\%), los Servicios e instituciones estatales (6,39\%), la Industria (6,24\%) y la Vivienda, construcción y patrimonio $(5,22 \%)$. La correlación con la agenda política fue Baja $(0,370)$, aunque los 9 ítems correlacionables se correspondían con la cantidad de menciones de este tipo en la agenda política.

Durante el cuarto período de tiempo solo 22 objetos tuvieron menciones positivas en la agenda mediática, entre los que se destacan la Salud pública $(12,42 \%)$, las Actividades políticas, institucionales y sociales $(11,74 \%)$, la Historia $(11,51 \%)$, la Industria $(9,03 \%)$, las Comunicaciones $(7,90 \%)$, las Organizaciones políticas y de masas $(7,67 \%)$ y la Educación $(7,45 \%)$. En este caso la correlación fue Alta $(0,632)$, con 4 elementos correlacionables de 5 que tenía la agenda política. 
Por otra parte, los objetos que tenían menciones neutras en la agenda mediática durante el primer período del estudio fueron las Actividades políticas, institucionales y sociales $(15,68 \%)$, los Problemas sociales $(12,89 \%)$, la Salud pública $(11,50 \%)$, las Comunicaciones (7,67\%), el Trabajo y seguridad social $(6,27 \%)$ y la Agricultura $(5,23 \%)$. Nótese la tendencia a tratar temas como la Salud Pública y los Problemas sociales desde una perspectiva neutra. En este caso la correlación con la agenda política fue Baja $(0,378)$, con 17 elementos correlacionables -los mismos que posee esta agenda en las menciones neutras de los objetos-. Esto quiere decir que aunque el valor haya dado bajo, todos los objetos de la agenda política con menciones neutras están en la agenda mediática, aunque puede que en otro orden de prioridad.

En el segundo período los objetos que más menciones neutras tenían fueron los Problemas sociales $(12,08 \%)$, la Salud pública (11,70\%), la Higiene comunal $(11,70 \%)$, el Transporte $(7,92 \%)$, las Actividades políticas, institucionales y sociales $(7,55 \%)$ y la Agricultura (6,79\%). La correlación en este caso fue Muy baja $(0,189)$, aunque los 10 objetos con estas menciones en la agenda política estuvieron en la agenda mediática, donde hubo 27 objetos.

Durante la tercera fase los objetos priorizados de forma neutra fueron la Salud pública $(15,18 \%)$, las Actividades políticas, institucionales y sociales $(13,53 \%)$, la Agricultura (8,25\%), los Servicios e instituciones estatales $(7,59 \%)$, y los Problemas sociales $(6,60 \%)$. La correlación con la agenda política fue Muy baja $(0,135)$, aunque 17 de los 19 objetos de la agenda política estuvieron mencionados también de forma neutra en la agenda mediática, donde hubo 24 objetos con menciones de este tipo.

Por último, en el cuarto período hubo 21 objetos con menciones neutras, entre los cuales se destacan las Actividades políticas, institucionales y sociales $(39,62 \%)$, la Salud pública (14,62\%), el Trabajo y seguridad social (9,43\%), el Acueducto (6,13\%), el Transporte $(4,72 \%)$ y la Historia $(4,25 \%)$. Nótese la ligera variación temática, aunque esta agenda no escapa del tratamiento neutral a asuntos socialmente demandados, como veremos posteriormente. La correlación con la agenda política fue Baja $(0,209)$, aunque de 23 objetos posibles en esta agenda, 20 fueron correlacionables con la agenda mediática, donde el valor es aún más cercano.

En cuanto a la dimensión negativa puede decirse que- a pesar de no poder establecer una correlación con la agenda política, por esta carecer de dichas menciones en su agenda- en la agenda mediática de CMKC sí hubo objetos con tratamiento negativo. En este caso fueron bajos los porcentajes del tratamiento negativo a estos temas. Por supuesto, siguen predominando las visiones positivas y neutrales en la agenda de este medio, como ocurrió en el caso anterior. Sin embargo es alentador que este medio aborde problemas de la sociedad y que están en la agenda pública de forma crítica- aunque la cifra no se significativa con respecto a las otras dimensiones afectivas-.

\section{CONCLUSIONES}

A modo de conclusión puede decirse que se cumple la hipótesis planteada al inicio del estudio, o sea, la agenda mediática de la emisora CMKC y la agenda política en Santiago de Cuba poseen una correlación significativa, particularidad que se 
mantiene constante en el tiempo estudiado. Las prioridades temáticas en la agenda política están relacionadas con las actividades como marchas, eventos, reuniones, entre otras, y otros temas como la Historia, la Vivienda y la Higiene. En tanto estas agendas tuvieron una correlación Moderada en los cuatro períodos analizados, resultado que es similar al obtenido por los autores Muñiz y Fonseca (2015).

Por otra parte, se evaluó la transferencia de atributos en la dimensión sustantiva y afectiva, encontrando en el primer caso que las correlaciones son Moderadas y Bajas, aunque con altos valores en los elementos correlativos. En el caso de las menciones positivas se halló que la relación es Alta en varios períodos de tiempo, lo que indica la transferencia de estas valoraciones de la agenda política hacia la agenda del medio. Lo contrario sucedió con las menciones neutras, donde a pesar de haber bajos resultados, pudimos ver que la mayoría de los objetos con estas menciones estuvieron presentes en ambas agendas, aunque en rangos diferentes.

A través del análisis de los datos puede decirse que la agenda política no solo transfiere en este caso los objetos, sino los temas específicos y las valoraciones, y que esta transferencia se mantiene constante en el período estudiado.

\section{REFERENCIAS}

\section{Libros completos:}

Gans, H. (1979) Deciding what's News: A Study of CBS Evening News, NBC Nightly News, Newsweek, and Time (Visions of the American Press). New York: Pantheon Books.

Gomis, L (1991) Teoría del periodismo. Cómo se forma el presente. España: Paidós Comunicación.

\section{Artículos en papel y electrónicos:}

Casermeiro, A. (2004). Los medios en las elecciones: la Agenda Setting en la Ciudad de Buenos Aires. Buenos Aires: Educa. Disponible en el URL: http:// bibliotecadigital.uca.edu.ar/repositorio/educa/medioseleccionesagenda.pdf Consultado el 30/10/2012.

Cook, F.L., Tyler, T.R., Goetz, E.G. et al (1983) Media and Agenda Setting: effects on the public, interest group leaders, policy makers, and Policy. Public Opinion Quarterly, pp. 47.

Dader, J.L. (1990), "La canalización o fijación de la "agenda" por los medios", en Muñoz, A. et al. Opinión pública y comunicación política, Madrid, Eudema Universidad, pp. 296-297.

Gautier, L. C., y Ruiz, C. D. M. (2014). El discurso político y su reflejo en los medios: la credibilidad en tiempos de crisis (2008-2011). Historia y Comunicación Social, 19, 129-137.

Goelzhauser, G. y Vouvalis N. (2015) “Amicus Coalition Heterogeneity and Signaling Credibility in Supreme Court Agenda Setting". En Publius, 45 (1), pp. 99-116. Estados Unidos: Oxford University Press. 
Jackman, M. (2014) "Parties, Median Legislators, and Agenda Setting: How Legislative Institutions Matter". En The Journal of Politics, volumen 76, enero 2014, pp 259-272.

Lindstädt, R., \& Vander Wielen, R. J. (2014). Dynamic Elite Partisanship: Party Loyalty and Agenda Setting in the US House. British Journal of Political Science, 44(04), 741-772.

López, P. y Casero A. (2014) La información periodística de portada en España: evolución histórica de fuentes, agendas y encuadres (1980-2010). Historia y Comunicación Social, Vol. 19. Nº Especial. Enero (2014) 457-473.

McCombs (2006) Estableciendo la agenda. El impacto de los medios en la opinión pública y el conocimiento. Barcelona: Paidós.

Muñiz, V. y Fonseca, R. (2015) Relación entre la agenda mediática de los órganos de prensa provinciales de Santiago de Cuba y la agenda política. Influencias en el proceso. Revista Santiago, No. 137 (mayo-agosto), pp. 316-346. Universidad de Oriente, Santiago de Cuba.

Petrone, F. (2009) Estudios de Agenda Setting. Conceptos, metodologías y abordajes posibles. (Material en PDF)

Porath, W. (2007) Los temas de la discusión pública en las elecciones presidenciales chilenas 2005: relaciones entre las agendas de los medios y las agendas mediatizadas de los candidatos y del gobierno. En América Latina Hoy, 46, pp. 41-73. España: Ediciones Universidad de Salamanca.

Roda, R. (1989), Medios de comunicación de masas. Su influencia en la sociedad y en la cultura contemporánea, Madrid, Centro de Investigaciones Sociológicas.

Rodríguez, R. (2004). Teoría de la Agenda-Setting. Aplicación a la enseñanza universitaria. Madrid: Observatorio Europeo de Tendencias Sociales.

Vara, A (2001) La influencia de los partidos políticos en la construcción de la agenda mediática y el rol de los periodistas como mediadores sociales. Aproximación teórico-práctica a las teorías de la agenda setting y de la agenda building. Tesis doctoral. Facultad de Comunicación, Universidad de Navarra, Pamplona.

Walgrave, S., Aelst, V., Thesen, G., Vliegenthart., R. (2014) Mediatization and the media's political agenda-setting influence. (Material en PDF).

Weaver, D.H., Graber, D.A., McCombs, M. y Eyal, C.H. (1982) Media agenda setting in a presidential election. Praeger, Nueva York. 